\title{
Segurança e Eficácia do Treinamento Físico na Insuficiência Renal Crônica
}

\section{Safety and Accuracy of Physical Training in Chronic Renal Insufficiency}

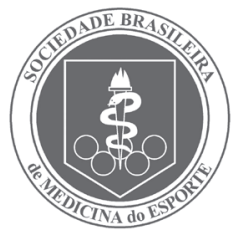

Artigo de ReVIsão
Cláudio Spínola Najas'

Flávio Danilo Mungo Pissulin

Francis Lopes Pacagnelli ${ }^{1}$

Gustavo Navarro Betônico²

Igor Costa Almeida²

José Alberto Neder ${ }^{3}$

1. Departamento de Fisioterapia, Setor de Fisioterapia em Pneumologia e Cardiologia do Hospital Universitário da Universidade do Oeste Paulista (Unoeste, Presidente Prudente, São Paulo).

2. Serviço de Nefrologia do Departamento de Clínica Médica da Faculdade de Medicina da Unoeste (Presidente Prudente, São Paulo).

3. Disciplina de Pneumologia, Departamento de Medicina, Universidade Federal de São

Paulo (Unifesp-EPM, São Paulo, São Paulo).

Trabalho realizado no Programa de Mestrado Profissionalizante do Laboratório de Fisiologia Respiratória (Lafirex) e do Exercício do

Departamento de Fisiologia da Unifesp-EPM.

\section{Endereço para correspondência:}

Cláudio Spínola Najas

Universidade do Oeste Paulista - Unoeste,

Departamento de Fisioterapia, Setor de

Fisioterapia em Pneumologia e Cardiologia do Hospital Universitário da Universidade do

Oeste Paulista

Rua José Bongiovani, 1.297, Vila Liberdade

19050-680 - Presidente Prudente, SP.

Tels. (18) 3229-1567 e (18) 3229-1362

E-mail:cnajas@gmail.com

Submetido em: 19/11/2008

Versão final recebida em: 10/05/2009

Aceito em: 14/05/2009

\section{RESUMO}

A doença renal crônica acarreta alterações em todos os sistemas corporais. Os pacientes cursam com marcada redução do condicionamento cardiorrespiratório, alterações musculares, reduzida performance física e pior qualidade de vida. A atividade física tem sido cada vez mais utilizada como forma de tratamento para essa população. Programas de treinamento físico aeróbio e/ou resistidos de moderada ou baixa intensidade no período interdialítico e durante a hemodiálise têm sido utilizados. Entretanto, os benefícios dessas intervenções no doente renal crônico, a escolha mais apropriada do tipo de treinamento e a segurança da aplicabilidade de atividades específicas não estão bem esclarecidos. Esta revisão tem como objetivo abordar os aspectos relacionados com o tipo de treinamento, período em que este é realizado, assim como os possíveis benefícios que o treinamento físico pode induzir nessa população.

Palavras-chave: atividade física, hemodiálise, metabolismo.

\begin{abstract}
Chronic renal disease has consequences on several physiological systems. Patients present decrease on cardiorespiratory capability, muscular changes, reduced physical performance and worse quality of life. Physical activity has been increasingly used as therapy for these patients, including training programs with aerobic and resistance exercises of low to moderate intensity in the interdialytic period and during hemodialysis. However, the benefits of physical exercise therapy to chronic renal patients, the choice of better training programs and the applicability safety of specific exercises are not clear yet. Thus, this review has the objective to discuss the aspects concerned with the type of training, better period to perform it and the relevance of their benefits to patients.
\end{abstract}

Keywords: physical activity, hemodialysis, metabolism.

\section{INTRODUÇÃO}

A insuficiência renal crônica (IRC) refere-se a um diagnóstico sindrômico de perda progressiva e habitualmente irreversível da função renal, caracterizada pela diminuição da taxa de filtração glomerular. A IRC associa-se a elevada morbimortalidade, levando, frequentemente, à incapacidade e redução apreciável da qualidade de vida. A existência de tratamentos paliativos de suporte, como o dialítico, prolonga substancialmente a sobrevida, tornando-a uma condição de impacto em longo prazo na saúde humana ${ }^{(1,2)}$.

A prevalência da IRC vem aumentando globalmente. Dessa forma, enquanto nos EUA há 336 casos/milhão de habitantes, na Europa encontram-se 135/milhão, com expectativa de crescimento anual de 5-8\%(4). No Brasil, em 2006, a incidência foi de 175 pacientes/milhão, representando aumento de 8,8\% em relação a 2005. Atualmente, estima-se que 54,5 mil pessoas encontram-se em tratamento dialítico, sendo 48,8 mil em hemodiálise. O número de pacientes em programa dialítico cresce no Brasil em média 10\%, devido à incidência de mais de 100 pacientes novos por milhão de habitantes/ano(5).

O diabetes mellitus é responsável por cerca de $45 \%$ dos novos casos de IRC nos Estados Unidos, 36\% na Alemanha, 32\% no Japão e aproximadamente 15\% em outros países europeus e na Austrália. Atualmente, no Brasil e na América Latina, cerca de 15\% dos indivíduos em diálise são diabéticos ${ }^{(6)}$.
No Brasil, dentre 2.467.812 pacientes com hipertensão e/ou diabetes cadastrados no programa HiperDia do Ministério da Saúde em 29 de março de 2004, a frequência de doenças renais foi de 6,63\% (175.227 casos), conforme a tabela 1.

Na IRC, os pacientes cursam com baixa tolerância ao exercício ${ }^{(7)}$. Também, a falta de prática de exercícios físicos por esses pacientes causa alterações musculoesqueléticas como fadiga e diminuição da resistência ${ }^{(7)}$. O sedentarismo nos indivíduos com IRC é consequência de um cotidiano monótono e limitado, principalmente após o início do tratamento, o que influencia diretamente na sua capacidade funcional(8).

Outros fatores que contribuem para a apresentação desse quadro são: baixo condicionamento físico, anemia, hipertensão arterial, dor e depressão(9).

Segundo a Diretriz de Reabilitação Cardiopulmonar e Metabólica (2006), tem sido demonstrada a importância da reabilitação, incluindo exercício físico, para nefropatas crônicos, inclusive os submetidos a programa de hemodiálise, os quais apresentam acentuada redução da capacidade cardiorrespiratória ${ }^{(10)}$. Pacientes nefropatas têm prevalência mais elevada de doenças cardiovasculares do que a população em geral, por isso, o treinamento físico é altamente benéfico ${ }^{(11)}$. Entretanto, há controvérsias quanto ao tipo de exercício, intensidade, duração, forma e local de realização mais adequados para esses pacientes. Dessa forma, o objetivo desta revisão é discutir a segurança do treinamento nessa 
Tabela 1. Fatores de risco para doença renal crônica

RISCO PARA DOENÇA RENAL CRÔNICA

\begin{tabular}{l|l}
\hline Elevado risco & $\begin{array}{l}\text { Hipertensão arterial } \\
\text { Diabetes mellitus } \\
\text { História familiar de DRC }\end{array}$ \\
\hline Médio risco & $\begin{array}{l}\text { Enfermidades sistêmicas } \\
\text { Infecções urinárias de repetição } \\
\text { Litíase urinária repetida } \\
\text { Uropatias } \\
\text { Crianças }<5 \text { anos } \\
\text { Adultos }>60 \text { anos } \\
\text { Mulheres grávidas }\end{array}$ \\
\hline
\end{tabular}

Doença Renal Crônica: Definiçăo, Epidemiologia e Classificação. João Egidio Romão Junior. J Bras Nefrol Volume XXVI - no 3 - Supl. 1 - Agosto de 2004

população, as modalidades de exercício utilizadas bem como os efeitos do exercício físico nesses pacientes.

\section{MECANISMOS DE INTOLERÂNCIA AO EXERCÍCIO NA IRC}

De acordo com Deligiannis ${ }^{(2)}$, os pacientes com insuficiência renal crônica em hemodiálise são caracterizados pela pequena tolerância em realizar exercícios e pelos sintomas de debilitação, mesmo com os avanços nos procedimentos de diálise e o uso da eritropoetina.

Segundo Kouidi ${ }^{(11)}$, há fatores centrais e periféricos que predispõem a uma redução da tolerância ao exercício nesse grupo.

Dentre os fatores centrais destacam-se as disfunções cardíacas e piora do controle cardíaco autonômico. Nessa população são muito comuns anormalidades cardiovasculares que incluem doença arterial coronariana, insuficiência cardíaca congestiva, arritmias e hipertensão. A hipertrofia ventricular esquerda resultante da cardiomiopatia urêmica é o achado morfológico mais comum, e esta cursa com disfunção sistólica e diastólica(11).

Quanto aos fatores periféricos estão as alterações do metabolismo oxidativo muscular, além de outras disfunções ${ }^{(11)}$. Especificamente a flexibilidade do músculo e a capacidade muscular diminuem, resultando em disfunção e atrofia musculoesquelética(4). Essas mudanças são presumidas, secundárias às toxinas urêmicas, anemia, desnutrição, descondicionamento, alterações neurormonais, entre outras. Estudos prévios têm demonstrado que as mudanças no limite do treinamento físico nos pacientes em hemodiálise ocorrem principalmente devido a alterações nos músculos esqueléticos dos membros. O impacto negativo da IRC no músculo esquelético deve-se a alterações também da perfusão muscular, uso de corticosteroide, estado catabólico mediado por vários fatores como acidose metabólica ${ }^{(4)}$.

Disfunções psicológicas como depressão em decorrência da doença renal e da inatividade são fatores relatados importantes que influenciam negativamente a função física nesses pacientes ${ }^{(12)}$.

\section{EXERCÍCIO FÍ́SICO E INSUFICIÊNCIA RENAL CRÔNICA TREINAMENTO FÍSICO NA IRC}

De acordo com Johansen ${ }^{(13)}$, pacientes com doença renal crônica são inativos e têm seu desempenho físico reduzido. Intervenções de treinamento aeróbico têm sido utilizadas para aumentar o consumo máximo de oxigênio em pacientes selecionados. Evidências preliminares sugerem que o treinamento aeróbico pode melhorar o controle da pressão arterial, perfil lipídico e saúde mental nessa população. Poucos estudos, porém amplos, estão agora disponíveis mostrando que o treinamento aeróbico pode também melhorar o desempenho físico, embora o impacto na hospitalização e sobrevivência não esteja bem determinado.
De outra forma, Johansen ${ }^{(14)}$ afirma que o treinamento de resistência, embora menos estudado, parece aumentar a flexibilidade, força e função muscular. Há vários relatos de intervenções de treinamentos combinados bem sucedidos. Porém, os resultados não permitem avaliação dos benefícios relativos do treinamento de resistência e aeróbico no desempenho físico. Embora existam evidências de que o exercício seja seguro e benéfico em pacientes renais crônicos, muitos pacientes em diálise permanecem inativos, pois esses programas não são frequentemente oferecidos.

Johansen ${ }^{(14)}$ afirma que a maioria dos benefícios conhecidos do treinamento para a população em geral são de relevância também para a população com doença renal crônica. O estudo das taxas da população renal crônica de 30 anos atrás e numerosas intervenções, incluindo treinamento aeróbico, treinamento de resistência e programa de treinamento combinados, têm mostrado efeitos benéficos. Recentemente, intervenções durante as sessões de hemodiálise têm-se tornado mais populares e mostrado ser os mais seguras.

Moinuddin e Leehey ${ }^{(15)}$ observaram em pacientes em hemodiálise submetidos a exercício aeróbico que estes apresentaram diminuição da resistência à insulina, decréscimo da gordura corporal e redução da microalbuminúria.

Esses mesmos autores demonstraram em pacientes com hemodiálise que a realização de exercícios resistidos aumenta força, função muscular e melhora do perfil metabólico (IGF).

Entretanto, Cheema et al. ${ }^{(16)}$ afirmaram que o exercício físico não é rotineiramente indicado para essa população quando comparado com pacientes com outras doenças crônicas.

Os riscos de exercícios nessa população não têm sido rigorosamente estudados, mas não tem havido nenhum relatório de problemas sérios resultantes da participação em um programa de treinamento com exercícios.

\section{Treinamento no período interdialítico}

Mustata et al. ${ }^{(17)}$ submeteram 11 pacientes com insuficiência renal crônica a um programa de condicionamento físico em esteiras ou bicicletas ergométricas, duas vezes semanais por três meses com 60 a $80 \%$ da FCmáx atingida em teste de esforço. Demonstraram que o treinamento provocou diminuição da rigidez arterial e da pressão de pulso, retornando aos valores anteriores após um mês de destreinamento, não ocorrendo alterações na resistência à insulina, demonstrando que os treinamentos de exercícios aumentam a força da parede arterial, diminuindo o fator de risco em pacientes com cateter venoso que estão em hemodiálise. O mesmo efeito ocorreu com os estudos de Leaf et al. ${ }^{(18)}$, que avaliaram em cinco pacientes com doença renal crônica, do sexo masculino, o efeito do exercício no tamanho da veia para a maturação das fístulas pelo método Cimino-Brescia. O treinamento foi realizado por seis semanas com exercícios para antebraço e braço não dominante constituídos de contrações isométricas da mão de 25 a 35\% de medida da capacidade venosa com tempo de 40 a 120 segundos. Como resultados, houve aumento significativo no tamanho da veia cefálica no braço exercitado comparado com o braço controle (dominante), medido na presença e ausência de torniquete; isso prova que uma simples resistência causa aumento no tamanho da veia cefálica, sendo este o responsável pela diminuição da morbidade do acesso vascular.

Kouidi et al. ${ }^{(19)}$ propuseram um programa de reabilitação para avaliar as alterações musculares em sete pacientes. O programa era de exercícios de 90 minutos, três vezes semanais durante seis meses. O treino consistia de aquecimento, exercícios aeróbicos, resistência de baixo peso, alongamento e desaquecimento com intensidade baseada na 
capacidade aeróbica máxima de cada paciente. Os resultados mostraram aumento na proporção das fibras tipo II e número de capilares e mitocôndrias; houve também aumento no $\mathrm{VO}_{2}$ de pico, no tempo do exercício e no pico de força das pernas direita e esquerda. Foi constatado também aumento na velocidade de condução nervosa de ambas as pernas, mostrando que o programa de reabilitação melhora a atrofia muscular, aumentando o desempenho global desses pacientes. Da mesma forma, Coelho et al. ${ }^{(1)}$ (2006) estudaram os efeitos do exercício nessa população em cinco pacientes com idade de $45 \pm 9$ anos, realizando três sessões semanais em um total de 24 sessões e submetidos a período de aquecimento, exercícios para membros superiores, bicicleta e esteira ergométrica, resfriamento, treino para musculatura flexora dos dedos e musculatura inspiratória. Foram utilizados testes de caminhada, ergométrico, manovacuometria e força da musculatura flexora dos dedos. Os resultados obtidos foram redução da PAS de repouso, melhora significativa da $\mathrm{Pi}_{\max }$ e $\mathrm{Pe}_{\max }$ do $\mathrm{VO}_{2 \max }$ e força da musculatura flexora das mãos, mostrando os benefícios nessa população, na medida em que melhorou seu condicionamento.

Koufaki et al. ${ }^{(20)}$ (2002) avaliaram os efeitos do treinamento aeróbico sobre a capacidade funcional em 33 pacientes aleatórios divididos em dois grupos (18 no grupo de exercícios e 15 no grupo controle). Os pacientes fizeram teste ergométrico e bicicleta para determinar o pico de $\mathrm{VO}_{2 \max }$ e o limiar ventilatório; a capacidade funcional foi avaliada usando medidas do teste sentar e levantar. O grupo exercício fez três meses de treino em bicicleta ergométrica três vezes semanais durante a hemodiálise. Os resultados encontrados mostraram adaptações periféricas após o período de treinamento, com melhora significativa da capacidade de pico do exercício desses pacientes, também ocorrido no estudo de Headley et al.(21); 10 pacientes estáveis foram submetidos durante 12 semanas a treinamento aeróbio e de resistência sobre força e habilidade funcional. As variáveis utilizadas foram gordura corporal, distância percorrida em teste, torque de pico do quadríceps, força máxima da mão, velocidade em caminhada normal e máxima e o tempo no teste sentar e levantar por 10 repetições. No treino de resistência foram utilizados pesos em circuito com 10 repetições e a escala de Borg, precedido de aquecimentos em cicloergômetro. Como resultados, houve aumento do $\mathrm{VO}_{2}$ de pico e limiar ventilatório. $\mathrm{OVO}_{2}$ cinético permaneceu inalterado com diminuição no tempo do teste sentar/levantar, aumento no torque de pico e da distância percorri- da em teste, indicando que esse período de treinamento aeróbico e resistência pode ser utilizado para melhora da força e capacidade funcional nessa população.

Konstantinidou et al.(22) realizaram três programas de reabilitação: um no período interdialítico, outro durante a hemodiálise e outro de exercícios não supervisionados (domiciliares), além do grupo controle. Avaliaram a capacidade aeróbica, o mais favorável e o preferido pelos pacientes. Os exercícios foram realizados durante 60 minutos, três vezes semanais por seis meses. No treino interdialítico foram realizados aquecimento, exercícios intermitentes e desaquecimento com intensidade de 60 a $70 \%$ da $\mathrm{FC}_{\max }$. Os exercícios dialíticos foram realizados duas horas iniciais da hemodiálise numa intensidade de $70 \%$ da $\mathrm{FC}_{\max }$ em bicicleta adaptada à cadeira por 30 minutos, exercícios de flexibilidade e força muscular por 30 minutos precedidos de cinco minutos de aquecimento e sucedidos por cinco minutos de desaquecimento. No treino domiciliar, realizaram exercícios de intensidade moderada, orientados a andar de bicicleta cinco vezes por 30 minutos numa intensidade de 50 a $60 \%$ da $\mathrm{FC}_{\max }$. Os resultados obtidos no treino interdialítico e dialítico foram o aumento no $\mathrm{VO}_{2 \max }$ de pico, do limiar anaeróbico e duração do exercício, sendo estes ocorridos em menores proporções durante a hemodiálise. Já o treino domiciliar e grupo controle não causaram alterações nos parâmetros mencionados, indicando que o programa de exercícios tem efeitos no aspecto clínico, na capacidade cardiorrespiratória, sendo em dias intercalados ao da hemodiálise a forma mais efetiva de treinamento e os exercícios durante a hemodiálise a forma mais preferível dos pacientes (tabela 2).

\section{TREINAMENTO NO PERÍODO DIALÍTICO}

Oh-Park et al. ${ }^{(23)}$ avaliaram 22 pacientes na faixa etária de 52 anos, três vezes semanais durante 31 semanas. Propuseram um programa de exercícios de força muscular e aeróbio no período de uma-duas horas iniciais da diálise. O exercício de força foi realizado com carga de 50\% da 1RM em três séries de 15 repetições de extensão de joelho; o treino aeróbio, em bicicleta adaptada durante 30 minutos não consecutivos (cinco minutos e descansa um) e a intensidade foi graduada pela percepção de esforço de Borg. Obtiveram resultados como aumento da força dos extensores do joelho, do condicionamento físico e na função mental inferida por questionário, mostrando assim que o programa de exercícios melhora a força muscular, função física, mental e possivel-

Tabela 2. Treinamento interdialítico

\begin{tabular}{|c|c|c|c|}
\hline Estudos & Número de pacientes & Intervenção (treinamento) & Resultados \\
\hline Mustata et al. $(2004)^{17}$ & 11 & $\begin{array}{l}\text { - Bicicleta/esteira } \\
2 \text { vezes semanais } \\
3 \text { meses }\end{array}$ & $\begin{array}{l}\text { - Diminuição da rigidez arterial e pressão de pulso } \\
\text { - Sem alterações na resistência à insulina }\end{array}$ \\
\hline Konstantinidou et al. $(2002)^{22}$ & 7 & $\begin{array}{l}3 \text { programas de reabilitação: período interdialítico, dialítico } \\
\text { e não supervisionado domiciliares, } 3 \text { vezes semanais por } \\
60 \text { min. }\end{array}$ & $\begin{array}{l}\text { Grupos dialíticos e interdialíticos, aumento do } \mathrm{VO}_{2 \max \text { }} \text { do limiar } \\
\text { anaeróbico e duração do exercício. } \\
\text { Treino domiciliar sem alterações. }\end{array}$ \\
\hline Leaf et al. $(2002)^{18}$ & 5 & $\begin{array}{l}6 \text { semanas de treinamento físico de antebraço. Contração } \\
\text { isométrica de mão, }\end{array}$ & Aumento do tamanho da veia cefálica. \\
\hline Headley et al. $(2002)^{21}$ & 10 & $\begin{array}{l}12 \text { semanas de treinamento de força para MMSS e II em } \\
\text { circuito com equipamentos. }\end{array}$ & $\begin{array}{l}\text { Aumento do pico de torque, da distância do teste de } 6 \text { min e velo- } \\
\text { cidade de caminhada. } \\
\text { Diminuição do tempo do teste sentar/levantar }\end{array}$ \\
\hline Kouidi et al. $(2004)^{12}$ & 48 & $\begin{array}{l}3 \text { vezes semanais por } 4 \text { anos. } \\
\text { Exercícios aeróbicos interdialíticos e aeróbicos dialíticos }\end{array}$ & $\begin{array}{l}\text { Aumento do } \mathrm{VO}_{2 \max } \text { da qualidade de vida e aptidão física e saúde } \\
\text { com proeminência no grupo interdialítico }\end{array}$ \\
\hline Kouidi et al. $(1998)^{19}$ & 7 & $\begin{array}{l}3 \text { vezes semanais por } 6 \text { meses. } \\
\text { Treino aeróbico (natação ou jogos com bola) }\end{array}$ & $\begin{array}{l}\text { Aumento na velocidade de condução nervosa, das fibras tipo II, do } \\
\mathrm{VO}_{2} \text { de pico, do tempo de exercício, no pico de força em MMII. }\end{array}$ \\
\hline
\end{tabular}


mente a capacidade cardíaca. Da mesma forma, Kouidi et al. ${ }^{(12)}$ realizaram estudo para avaliar o efeito de dois programas de treinamento em longo prazo. Os parâmetros analisados foram aptidão física, percepção de saúde e condições gerais de vida, sendo avaliados 48 pacientes com período de exercícios de quatro anos divididos em dois grupos: um com exercícios domiciliares não supervisionados e outro em bicicleta estacionária durante a hemodiálise três vezes semanais em ambos os grupos. A aptidão física foi medida por protocolo de Bruce modificado em esteira e estudo ergoespirométrico; a percepção de saúde e as condições gerais de vida, através de questionário autoaplicativo. Obtiveram como resultados em relação à aptidão física aumento significativo nos dois grupos, principalmente no primeiro, melhora da percepção de saúde e situação global de vida; a percepção de melhora foi maior no primeiro grupo. Foi assim observado que pacientes em hemodiálise podem aderir a programas em longo prazo supervisionados ou não para obter melhora na aptidão física e saúde.

Macdonald et al. ${ }^{(24)}$ realizaram estudo com objetivo de avaliar se um programa de exercícios dialítico contribui para o ganho de massa muscular por intermédio do fator de crescimento semelhante à insulina (IGF). Participaram nove pacientes durante três meses em um programa de treinamento intervalado usando exercícios de alta intensidade. Para avaliação do IGF foram coletados biópsia muscular e sangue, a força estática do quadríceps por meio do dinamômetro e procedimento de imagem para avaliar a massa muscular. Após o treinamento, foi observado aumento no pico de potência máxima na carga de treinamento e capacidade física, enquanto as concentrações sanguíneas e musculares de IGF e a massa muscular permaneceram inalteradas. Diante disso se constata que 12 semanas de treino de alta intensidade melhoram a capacidade física, porém não revertem a atrofia. Tal resposta não ocorreu no estudo de Storer et al. ${ }^{(7)}$, onde avaliaram 20 pacientes e propuseram um treino de endurance em cicloergômetro adaptado à cadeira de diálise a $50 \%$ do $\mathrm{VO}_{2 \max }$ a uma tolerância máxima de carga de trabalho, três vezes semanais por nove semanas. Houve melhora na função cardiopulmonar, na força muscular de membros inferiores, na potência muscular, na fadigabilidade e no tempo de endurance; é indicativo de que nove semanas de treino de endurance melhoram não somente a capacidade e a condição cardiopulmonar, mas também a força muscular, fadigabilidade e função física.

Kong et al. ${ }^{(25)}$ estudaram o efeito dos exercícios na remoção da ureia, creatinina e potássio. Foram avaliados 11 pacientes de 32 a 78 anos com tempo de hemodiálise de quatro a 58 meses. Todos foram analisados em duas sessões de hemodiálise com idêntica prescrição em semanas consecutivas e submetidos a exercícios de pedalar de cinco a 20 minutos em trabalho submáximo com 10 minutos de descanso para um total de 60 minutos de exercício. As concentrações de ureia, creatinina e potássio foram medidas pré-dialítico, imediatamente após e 30 minutos pós-diálise. Foi observada redução significativa dos três solutos após os exercícios, mostrando assim que o exercício aumentou a eficiência dialítica pela redução do rebote dos solutos devido ao aumento da perfusão dos músculos esqueléticos.

Painter et al.(26) avaliaram em 286 pacientes o efeito de dois programas de exercícios sobre níveis de atividade física, capacidade física e autorrelato sobre seu estado de saúde. As intervenções foram individuais e os exercícios foram domiciliares não supervisionados por oito semanas, seguidos por oito semanas supervisionados em bicicleta ergométrica durante a hemodiálise; para os dois grupos foi utilizado exercício aeróbico. Os testes para avaliação física foram a marcha, o teste de caminhada e o teste sentar/levantar. O questionário SF36 foi utilizado para autoavaliação de qualidade de vida. Todos os grupos que realizaram exercícios melhoraram em todos os testes realizados, principalmente nos exercícios não supervisionados, ressaltando que a melhora no funcionamento físico resulta de orientação do exercício e encorajamento aos pacientes. Da mesma forma, os mesmos autores, Painter et al.(27), compararam a resposta da intervenção de pacientes que inicialmente tinham um baixo escore no SF-36 (menor que 34) com aqueles que possuíam um alto escore (maior que 34). O funcionamento físico e a qualidade de vida foram avaliados após dois meses de exercícios não supervisionados e depois de dois meses de bicicleta estacionária adaptada para a diálise. Os exercícios não supervisionados foram de força, flexibilidade e condicionamento cardiovascular A intensidade do esforço nos grupos foi determinada pela escala de percepção de esforço modificada. Foi avaliada pelo teste sentar e levantar, marcha normal e marcha rápida. Os resultados demonstraram que o grupo que tinha o escore maior no SF-36 melhorou no teste de sentar/levantar, enquanto o grupo com menor escore no SF-36 melhorou nos três testes de capacidade física, sendo concluído que os pacientes de hemodiálise com baixa capacidade física, podem se beneficiar de exercícios assistidos. $\mathrm{O}$ impacto de tais intervenções parece ser de maior magnitude em pacientes com maior comprometimento da função renal (tabela 3).

Tabela 3. Treinamento dialítico.

\begin{tabular}{|c|c|c|c|}
\hline Estudos & Número de pacientes & Intervenção (exercícios) & Resultados \\
\hline Oh-Park et al. $(2002)^{23}$ & 18 & 3 vezes semanais por 21 semanas, exercício aeróbico e força muscular. & $\begin{array}{l}\text { Aumento da força de extensão do joelho, melhora do } \\
\text { condicionamento físico e função mental. }\end{array}$ \\
\hline Storer et al. $(2005)^{7}$ & 20 & 3 vezes semanais por 9 semanas, treinamento de endurance. & $\begin{array}{l}\text { Aumento da função cardiopulmonar, potência, força e } \\
\text { fadigabilidade muscular. }\end{array}$ \\
\hline Kong et al. $(1999)^{25}$ & 11 & Sessão única de exercício em ciclo ergômetro por 60 min. & $\begin{array}{l}\text { Aumento do KtV (eficiência dialítica), diminuição da crea- } \\
\text { tinina, potássio, efeito rebote da ureia. }\end{array}$ \\
\hline Painter et al. $(2000)^{27}$ & 286 & $\begin{array}{l}3 \text { a } 4 \text { vezes semanais exercícios de caminhada, flexibilidade e força. } \\
\text { Exercício domiciliar não supervisionado } \\
\text { Exercício durante hemodiálise em bicicleta ergométrica. }\end{array}$ & $\begin{array}{l}\text { Aumento da distância percorrida, da velocidade de mar- } \\
\text { cha, da qualidade de vida, menor tempo no teste sen- } \\
\text { tar/levantar }\end{array}$ \\
\hline Macdonald et al. $(2005)^{24}$ & 9 & $\begin{array}{l}3 \text { meses de treinamento intervalado de alta intensidade (exercício de } \\
\text { resistência para hipertrofia) }\end{array}$ & $\begin{array}{l}\text { Aumento da capacidade física e não revertida a atrofia } \\
\text { muscular. }\end{array}$ \\
\hline Coelho et al. (2006) ${ }^{1}$ & 5 & $\begin{array}{l}3 \text { vezes semanais nos dias da diálise. } \\
\text { Treino aeróbico bicicleta/esteira. } \\
\text { Treino muscular específico (músculos inspiratórios e flexores dos dedos) }\end{array}$ & $\begin{array}{l}\text { Aumento da Pl e } \mathrm{PE}_{\max \prime} \text { da força da musculatura flexora das } \\
\text { mãos e aumento do } \mathrm{VO}_{2} \text {. } \\
\text { Diminuição da PAS. }\end{array}$ \\
\hline
\end{tabular}




\section{SEGURANÇA DURANTE UM PROGRAMA DE EXERCÍ- CIOS EM INDIVÍDUOS COM INSUFICIÊNCIA RENAL CRÔNICA}

Banerjee et al. ${ }^{(3)}$ demonstraram que um programa de exercício durante a hemodiálise provoca alterações hemodinâmicas semelhantes às que ocorrem em indivíduos saudáveis. Foram dois protocolos em bicicleta estacionária, com intensidade da FC 20\% maior que a de repouso. Em um deles foram dois períodos de 10 minutos separados por 10 minutos de repouso, enquanto no outro foram 10 minutos contínuos. $O$ débito cardíaco aumentou de 4,7 para 9,9L/min no primeiro protocolo e de 5,1 para 10,3L/min no segundo protocolo. O volume sanguíneo relativo diminuiu para $97 \%$ ao final do primeiro protocolo e para $96 \%$ ao final do segundo protocolo de exercício. O volume sistólico aumentou de 54,3 para $88,3 \mathrm{~mL}$ no primeiro protocolo e de 58,6 para $92,1 \mathrm{~mL}$ no segundo protocolo. Foi concluído que a resposta hemodinâmica para exercícios durante a hemodiálise nos renais crônicos pode ser comparada com aquela de indivíduos normais. A rápida redução no volume relativo sanguíneo em exercícios ocorre apesar de um significante aumento no batimento cardíaco, principalmente como consequência do deslocamento de fluído da microcirculação para o interstício.

\section{REFERÊNCIAS BIBLIOGRÁFICAS}

1. Coelho DM, Castro AM, Tavares HA, Abreu PCB, Glória RR, Duarte MH, et al. Efeitos de um programa de exercícios físicos no condicionamento de pacientes em hemodiálise. J Bras Nefrol. 2006;28:121-7.

2. Deligiannis A. Exercise rehabilitation and skeletal muscle benefits in hemodialysis patients. Clin Nephrol. 2004;61:46-50.

3. Banerjee A, Kong CH, Farrington K. The haemodynamic response to submaximal exercise during isovolaemic haemodialysis. Nephrol Dial Transplant. 2004;19:1528-32.

4. Adams GP, Varizi ND. Skeletal muscle disfunction in chronic renal failure: effects of exercise. Am J Physiol Renal. 2006;290:753-61.

5. Busato O. Insuficiência Renal (IR). Rio Grande do Sul, 2001. Disponível em: http://www.abcdasaude. com.br/artigo.php?266. acessado em [2005 Agosto 03].

6. Peres LAB, Matsuo T, Delfino VDA, Peres CPA, Almeida Netto JH, Ann HK, et al. Aumento na prevalência de diabete melito como causa de insuficiência renal crônica dialítica: análise de 20 anos na região Oeste do Paraná. Arq Bras Endocrinol Metabol. 2007;51:111-5.

7. Storer TW, Casaburi R, Sawelson S, Kopple JD. Endurance exercise training during haemodialysis improves strength, power, fatigability and physical performance in maintenance haemodialysis patients. Nephrol Dial Transplant. 2005;20:1429-37.

8. Martins MRI, Cesarino CB. Qualidade de vida de pessoas com doença renal crônica em tratamento hemodialítico. Revista Latino-Americana de Enfermagem. 2005;13:670-6.

9. Medeiros MMC, Holanda FFN, Mota, RS, Patrocínio RMV, Conrado FM, Lima GRM. Análise de 95 biópsias renais de pacientes com nefrite lúpica: correlação clínico-histológica e fatores associados a insuficiência renal crônica. Rev Bras Reumatol. 2004;44:268-76.

10. Diretriz de reabilitação cardiopulmonar e metabólica: aspectos práticos e responsabilidades. Arq Bras Cardiol. 2006;86:74-82.

11. Kouidi EJ. Central e Peripheral Adaptations to Physical Training in Patients with End-Stage Renal Disease Sports Med. 2001;31:651-65.

12. Kouidi E, Grekas D, Deligiannis A, Tourkantonis A. Outcomes of long-term exercise training in dialysis patients: comparison of two training programs. Clin Nephrol. 2004;61:31-8.

13. Johansen KL. Exercise and chronic kidney disease. Sports Med. 2005;35:485-99.

14. Johansen KL. Exercise in the end-stage renal disease population. J Am Soc Nephrol. 2007;18:1845-54.
A segurança do treinamento interdialítico nessa população deve estar relacionada com adequações da medida da eficiência dialítica $(\mathrm{Kt} / \mathrm{N})>1$, hemoglobina $>10 \mathrm{~g} / \mathrm{dL}$, ganho de peso interdialítico $<2,5 \mathrm{~kg}$ e potássio pré-dialítico $<5,5 \mathrm{mmol} / \mathrm{I}^{(20,28)}$. Um adequado controle das patologias de base também precisa ser considerado.

Quando o treinamento for realizado durante a hemodiálise, o controle adequado da presença de sinais cardiovasculares como dispneia, tontura, dor torácica e sinais vitais, ou seja, estabilidade hemodinâmica, deve ser considerado(24).

Programas de treinamento de resistência e aeróbico devem ser iniciados em baixa intensidade e com uma progressão lenta de acordo com a tolerância do paciente.

\section{CONCLUSÃO}

Conclui-se que a atividade física assim como os programas de treinamentos aeróbicos, resistidos e combinados, beneficiam os pacientes renais crônicos, tanto nas fases dialíticas como interdialíticas. Os programas de treinamento e exercícios são propostas não farmacológicas seguras e eficazes para essa população, tendo efeitos incrementais na capacidade cardiorrespiratória, condicionamento físico, força muscular, capacidade funcional, consumo de oxigênio máximo $\left(\mathrm{VO}_{2 \max }\right)$, eficiência dialítica, redução dos solutos e resposta hemodinâmica.
15. Moinuddin I, Leehey DJ. A Comparison of Aerobic Exercise and Resistance Training in Patients With and Without Chronic Kidney Disease. Adv Chronic Kidney Dis. 2008;15:83-96.

16. Cheema B, Abas H, Smith B, O'Sullivan A, Chan M, Patwardhan A, et al. Randomized Controlled Trial of Intradialytic Resistance Training to Target Muscle Wasting in ESRD: The Progressive Exercise for Anabolism in Kidney Disease (PEAK) Study. Am J Kidney Dis. 2007;50:574-84.

17. Mustata S, Chan C, Lai V, Miller JA. Impact of an exercise program on arterial stiffines an insulin resistance in hemodialysis patients. J Am Soc Nephrol. 2004;15:2713-8.

18. Leaf DA, Holden SH, Grant E, Kraut J. Isometric Exercise increases the size of forearm veins in patients with chronic renal failure. Am J Med Sci. 2003;325:115-9.

19. Kouidi E, Albani M, Natsis K, Megalopoulos A, Gigis P, Tziampiri OG, et al. The efects of exercise training on muscle atrophy in haemodialysis patients. Nephrol Dial Transplant. 1998;13:685-99.

20. Koufaki P, Mercer TH, Naish PF. Effects of exercise training on aerobic an functional capacity of end-stage renal disease patients. Clin Physiol Funct Imaging. 2002;22:115-24.

21. Headley S, Germain M, Mailloux P, Mulhern J, Ashworth B, Burris J Brewer B, Nindl B, Coughlin M, Welles $R$, Jones $M$. Resistance training improves strength an functional measures in patients with end-stage renal disease. Am J Kidney Dis. 2002;40:355-64.

22. Konstantinidou E, Koukouvou G, Kouidi E, Deligiannis A, Tourkantonis A. Exercise training in patients with end-stage renal disease on hemodialysis: Comparison of Three Rehabilitation Programs. J Rehabil Med. 2002;34:40-5.

23. Oh-Park M, Fast A, Gopal S, Lynn R, Frei G, Drenth R, et al. Exercise for the dialized: aerobic and strength training during hemodialysis. Am J Phys Med Rehabil. 2002;81:814-21.

24. Macdonald JH, Marcora SM, Jibani M, Phanish MK, Holly J, Lemmey AB. Intradialytic exercise as anabolic therapy in haemodialysis patients - a pilot study. Clin Physiol Funct Imaging. 2005;113-8.

25. Kong CH, Tattersall JE, Greenwood RN, Farrington K. The effect of exercise during haemodialysis on solute removal. Nephrol Dial Transplant. 1999;14:2927-31

26. Painter P, Carlson L, Carey S, Paul SM, Myll J. Physical functioning and health-related quality-of-life changes with exercise training in hemodialysis patients. Am J Kidney Dis. 2000;35:482-92.

27. Painter P, Carson L, Carey S, Paul SM, Myll J. Low-functioning hemodialysis patients improve with exercise training. Am J Kidney Dis. 2000;36:600-8.

28. Mercer TH, Crawford C, Gleeson NP, Naish PF. Low-volume exercise rehabilitation improves functional capacity an self-reported functional status of dialysis patients. Am J Phys Med Rehabil. 2002;81:162-7. 